\title{
In situ magnetorheological SANS setup at Institut Laue-Langevin
}

\author{
Dominika Zákutná $^{1,2,3}$ (D) Kevin Graef $^{1} \cdot$ Dominique Dresen $^{1}$ (D) $\cdot$ Lionel Porcar ${ }^{2} \cdot$ Dirk Honecker $^{2,4}$ (D) \\ Sabrina Disch ${ }^{1}$ (1)
}

Received: 9 April 2020 / Revised: 30 June 2020 / Accepted: 21 July 2020 / Published online: 3 September 2020

(C) The Author(s) 2020

\begin{abstract}
A magnetorheological sample environment is presented that allows for in situ magnetic field and shear flow during small-angle neutron scattering (SANS) measurements and is now available at the Institut Laue-Langevin (ILL). The setup allows performing simultaneous magnetorheological measurements together with the investigation of structural and magnetic changes on the nanometer length scale underlying the rheological response of ferrofluids. We describe the setup consisting of a commercial rheometer and a custom-made set of Helmholtz coils and show exemplarily data on the field and shear flow alignment of a dispersion of hematite nanospindles in water.
\end{abstract}

Keywords Small-angle neutron scattering $\cdot$ Magnetorheology $\cdot$ Rheology $\cdot$ Magnetoviscous effect $\cdot$ Magnetic nanoparticles

\section{Introduction}

Recent progress toward magnetic soft matter combines the unique properties of soft matter and magnetic materials into complex magnetic fluids, such as original or inverse ferrofluids, ferrogels, and ferroelastomers [1-9]. Complex magnetic fluids find technological application in vibro-protection, lubrication, and for the precise control of polishing processes $[10,11]$, actuators and sensors such as shapeprogrammable matter [12], as well as the field-controlled release of incorporated drugs [13-15]. Ferromagnetic liquid crystals combine nematic order as well as ferromagnetic order and show non-Newtonian behavior in flow field [16-19]. Magnetic nanorod dispersions have been further demonstrated as in situ and non-contact viscosity gauges,

Sabrina Disch

sabrina.disch@uni-koeln.de

1 Department für Chemie, Universität zu Köln, Greinstraße 4-6, 50939 Köln, Germany

2 Institut Laue-Langevin, 71 Avenue des Martyrs, F-38042 Grenoble, France

3 Present address: Department of Inorganic Chemistry, Faculty of Science, Charles University in Prague, Hlavova 2030/8, 12843 Prague 2, Czech Republic

4 Present address: Physics and Materials Science Research Unit, University of Luxembourg, 162A Avenue de la Faïencerie, L-1511 Luxembourg, Grand Duchy of Luxembourg being suitable probes for active microrheology [20]. The rotational friction of magnetic nanorods is sensitive to the stiffness of hydrogels [21] or the viscosity of liquid samples [22]. Recording the concomitant orientational redistribution with AC susceptibility, optical transmission spectroscopy [23], depolarized dynamic light scattering [24], SAXS [25], or SANS [26] enables to extract hydrodynamic properties of the carrier medium.

Magnetic fluids consist of magnetic particles suspended in a non-magnetic carrier liquid. With the assistance of a magnetic field, the dynamics and hence the flow properties of the dispersion can be tuned. The fluid-mechanical properties are determined by the particle orientation and the potential creation of clusters, where the interparticle arrangement is governed by the competition of attractive magnetic dipolar and disruptive shear forces. The knowledge of such flowand field-induced microstructural changes is important to understand the origin of the magnetorheological properties, including the magnetoviscous effect and shear-thinning and shear-thickening behaviors.

For dilute ferrofluids consisting of spherical particles, the magnetoviscous effect is understood as the field-induced hindrance of the free particle rotation in a shear flow leading to a small increase in viscosity [27]. This effect is enhanced for dilute, elongated nanoparticles in the liquid carrier. In the absence of magnetic field and neglecting Brownian thermal fluctuations, the motion of spheroidal particles in shear flow was solved analytically by Jeffery [28]. Particles suspended in a viscous fluid rotate about the flow vorticity vector. In shear 
flow, prolate spheroids will tumble and in the absence of inertial effects the rotation will follow so-called Jeffery orbits.

When a magnetic field is applied, anisometric magnetic nanoparticles align with their magnetic easy axis along the field [29-36]. If the long nanoparticle axis aligns perpendicular to the flow direction, the aligned nanoparticles oppose resistance to the flow resulting in an increase fluid viscosity. In contrast to common ferromagnetic nanorods, the easy magnetic axis of hematite spindles lies in the basal plane (i.e., the equatorial direction) such that the particles typically orient with their long axis perpendicular to an applied field [30, 32-34] with the peculiar results that a negative magnetorheological effect is observed in a magnetic field oriented perpendicular to the shear direction [37]. Hence, the application of magnetic field allows either enhancing alignment or overcoming shear alignment of magnetic fibers during sheardominated processing techniques [38].

With increasing particle volume fraction, enhanced magnetic dipolar interparticle interactions may lead to nanoparticle arrangements, e.g., chains, rings, and hexagonal columns as observed by small-angle scattering [39, 40]. In modest magnetic fields, the (reversible) formation of superstructures gives eventually rise to a significant increase of the viscosity by several orders of magnitude [41]. This effect is based on the magnetic field-induced alignment of the superstructures perpendicular to the flow direction [42]. These superstructures collapse already at moderate shear rates, which is the cause of the commonly observed shear-thinning behavior.

These examples illustrate that the viscosity reflects an adaption in the microstructure, superstructure formation, or particle reorientation, demonstrating the importance to observe in situ microstructural changes in the dispersion under magnetic and flow fields to find correlation with the fluidmechanical response.

Small-angle scattering techniques using X-ray and neutron radiation are versatile tools to obtain information on microstructural modifications on the nanometer length scale. Nowadays, rheometers are standard sample environments to investigate flow-induced effects on the mesoscopic scale from 1 to several hundred nanometers using small-angle scattering [43-54]. However, the combination of magnetic field and shear flow for small-angle neutron scattering is rare [53-57].

Here we present first results of a specialized sample environment for in situ MagnetoRheoSANS that is available to the wide scientific community through the user program at the Institut Laue-Langevin (ILL). It consists of an Anton Paar rheometer MCR501, which exists at the Institut LaueLangevin to perform rheological experiments together with simultaneous SANS, and a custom made shielded magnetic coil set, which provides a static magnetic field of up to $20 \mathrm{mT}$. This setup allows investigating the structure and magnetic configuration within a ferrofluid under magnetic field and shear flows providing information on the connection between rheological properties and the underlying microstructural response on complex magnetic fluids. As a proof of concept, MagnetoRheoSANS data of hematite nanospindles are presented.

\section{Materials and methods}

A set of Helmholtz coils was constructed to fit the existing Anton Paar rheometer MCR501 and provide a homogeneous magnetic field in the sample volume, perpendicular to the shear direction. The coil set has an outer diameter of $192 \mathrm{~mm}$. The coils are made of copper wire with a diameter of $1.48 \mathrm{~mm}$ and 820 windings each, generating a practically usable maximum field of $20 \mathrm{mT}$ in the center of the setup (Fig. 1). According to simulations (Fig. 1c-d), the field homogeneity at the sample position in the rheology cup is expected better than $0.3 \%$ over the SANS measurement position $(5-\mathrm{mm}$ height, red box in Fig. 1d) and better than $6 \%$ for the rheology measurement gap region (35-mm height, gray box in Fig. 1d). With a distance of $141 \mathrm{~mm}$ between the top and bottom of the Helmholtz coil set, an optical free distance of $35 \mathrm{~mm}$ in height allows for a maximum scattering angle of $2 \theta=10^{\circ}$. The coils can be cooled by water, ethylene glycol, or nitrogen stream in order to maintain a stable temperature during the measurement. A cup for Mooney Ewart rheological geometry has been specially constructed by Anton Paar made of titanium with an extended socket of $60 \mathrm{~mm}$ in height (Fig. 1b). The bottom of the socket is $54 \mathrm{~mm}$ wide and fits in the center of the magnetic coils. The measurement part has a diameter of $30 \mathrm{~mm}$ and a height of $35 \mathrm{~mm}$, such that the measurement gap is situated in the homogeneous magnetic field between the two coils, indicated in Fig. 1c, d. Soft magnetic iron plates shield the rheometer drive against the stray field of the coils. For a bob diameter of $29 \mathrm{~mm}$ the shear gap of $0.5 \mathrm{~mm}$, corresponding to a neutron path length of $1 \mathrm{~mm}$ in transmission geometry, requires a sample volume of $2.6 \mathrm{~mL}$.

A practically usable maximal magnetic field of $20 \mathrm{mT}$ was achieved at the position of the sample in the neutron beam, whereas for higher magnetic fields, we observed significant heat dissipation from the magnetic coils. Experiments using a higher field of $30 \mathrm{mT}$ led to an increase of the temperature by 4 degrees (measured at the temperature sensor at the coils) within 15 min of operation, accompanied by an increased coil resistance. Whereas the sample temperature in the rheology cup may in principle be stabilized using a dried air stream (not implemented in our experiment), the increasing coil resistance will have a direct impact on the generated magnetic field. Therefore, a maximal, reliable applied field of $20 \mathrm{mT}$ is recommended for our setup. The specifications of the magnetic coil set are summarized in Table 1.

As test sample, we used spindle-shaped hematite nanoparticles presented and precharacterized recently [30]. The 
Fig. 1 a Design of the Helmholtz coils with $\mathbf{b}$ a technical sketch of the measurement cup and c-d simulation of the magnetic field homogeneity. Data in (d) represent the local field homogeneity at the sample position in the cup at $x=15 \mathrm{~mm}$ (compare with (c)) with the black and red rectangles indicating the cup and beam dimensions, respectively (a)
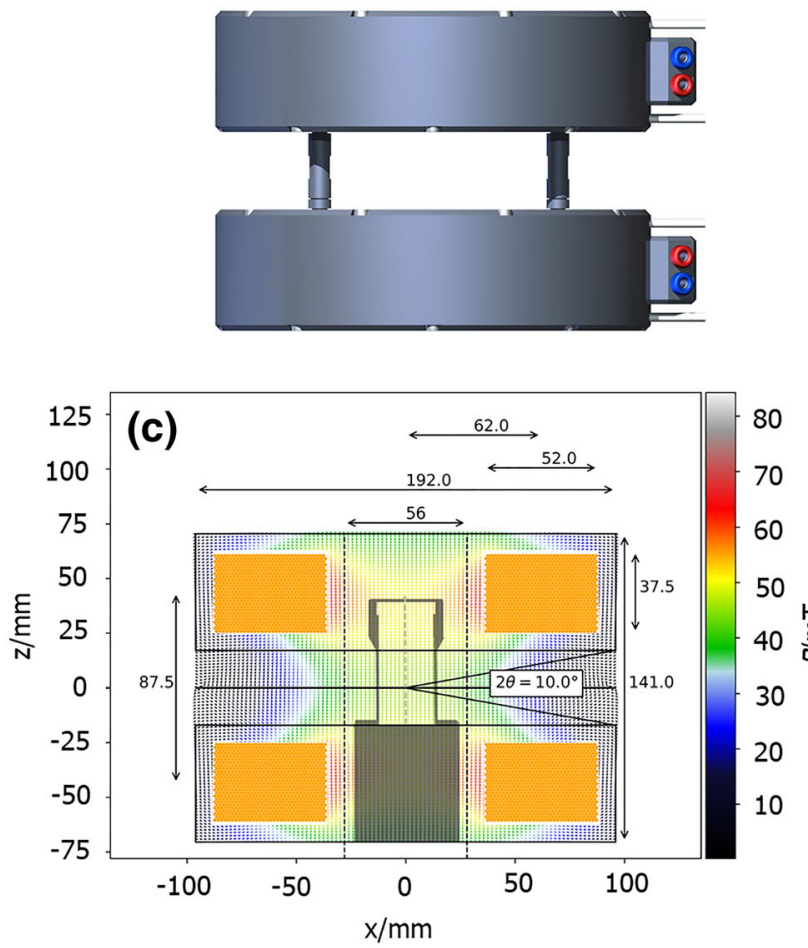

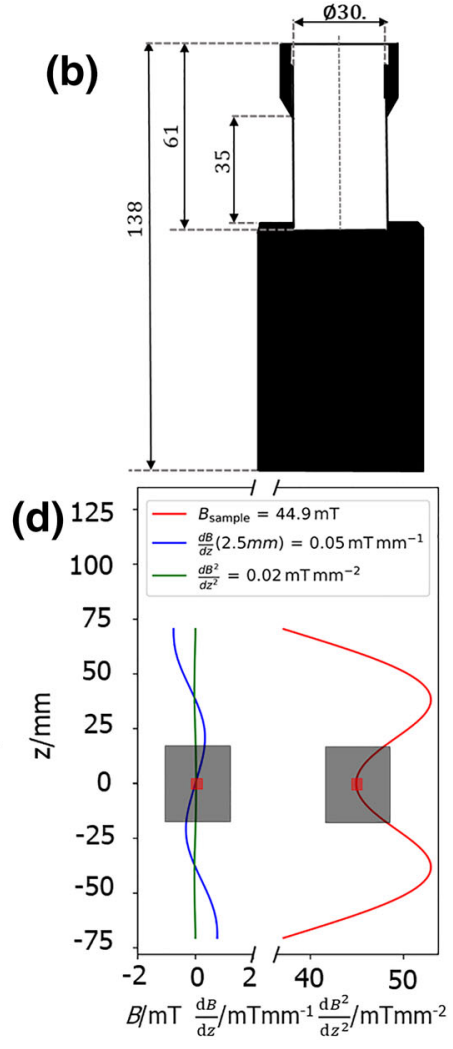

known and well-defined orientation behavior in applied magnetic field makes them a suitable model sample for our MagnetoRheoSANS setup. In brief, hematite nanospindles

Table 1 Technical parameters of the Helmholtz coils used for MagnetoRheoSANS

\section{Dimensions}

Height (mm)

Outer diameter $(\mathrm{mm})$

Inner diameter $(\mathrm{mm})$

Optical free distance (mm)

Wire diameter $(\mathrm{mm})$

Maximum scattering angle $2 \theta\left(^{\circ}\right)$

Magnetic field

Maximal magnetic field (mT)*

Field homogeneity (\%)

Technical parameters of cup

Total height (mm)

Height of socket (mm)

Diameter of the socket (mm)

Optically free path (mm)

Inner diameter $(\mathrm{mm})$

Bob diameter (mm)

*Where field stability is not affected by energy dissipation

**In our experiment, a bob with $29 \mathrm{~mm}$ diameter was used were synthesized by the hydrolysis technique according to Matijević and co-workers [58]. The nanospindles are characterized by a particle length of 375(14) nm and an equatorial diameter of 56(2) nm with a lognormal size distribution in both dimensions of $\sigma_{\log }=19 \%$, as well as an aspect ratio of 6.7(1) [30]. The integral moment of the particle determined by magnetometry is $\mu=6.6(1) \cdot 10^{-19} \mathrm{~J} \mathrm{~T}^{-1}$, equivalent to 7.1(1) $10^{4} \mu_{\mathrm{B}}$ with a spontaneous magnetization of $\sigma_{\mathrm{S}}=1.566(1) \mathrm{kA}$ $\mathrm{m}^{-1}$. The spindles have a magnetic volume of $V_{\text {mag }}=\mu / \sigma_{\mathrm{S}}=$ $4.2(1) \cdot 10^{-22} \mathrm{~m}^{3}$, which is slightly smaller than the structural particle volume of $\mathrm{V}_{\mathrm{TEM}}=5.0(3) \cdot 10^{-22} \mathrm{~m}^{3}$. For MagnetoRheoSANS experiments, the nanospindles were dispersed in water with a concentration of 3 vol\%. The low concentration was chosen in order to minimize the magnetic field effect on the rheological properties of the sample and to show the individual effects of field- and shear-induced nanoparticle orientations.

The first MagnetoRheoSANS experiment using the setup was conducted at D22 instrument at ILL, Grenoble, France [59]. SANS experiments were performed with a neutron wavelength of $6 \AA$ and a sample-to-detector distance of $17.6 \mathrm{~m}$ with symmetrical collimation setting. The Anton Paar MCR501 rheometer together with the Helmholtz coils was installed at the sample position (Fig. 2). The measurements were done in Mooney Ewart geometry, applying vertical magnetic fields of 0-20 mT strength and shear rates increasing stepwise from 0 to $600 \mathrm{~s}^{-1}$ using the rotational mode of the rheometer. The applied magnetic field is parallel to the 


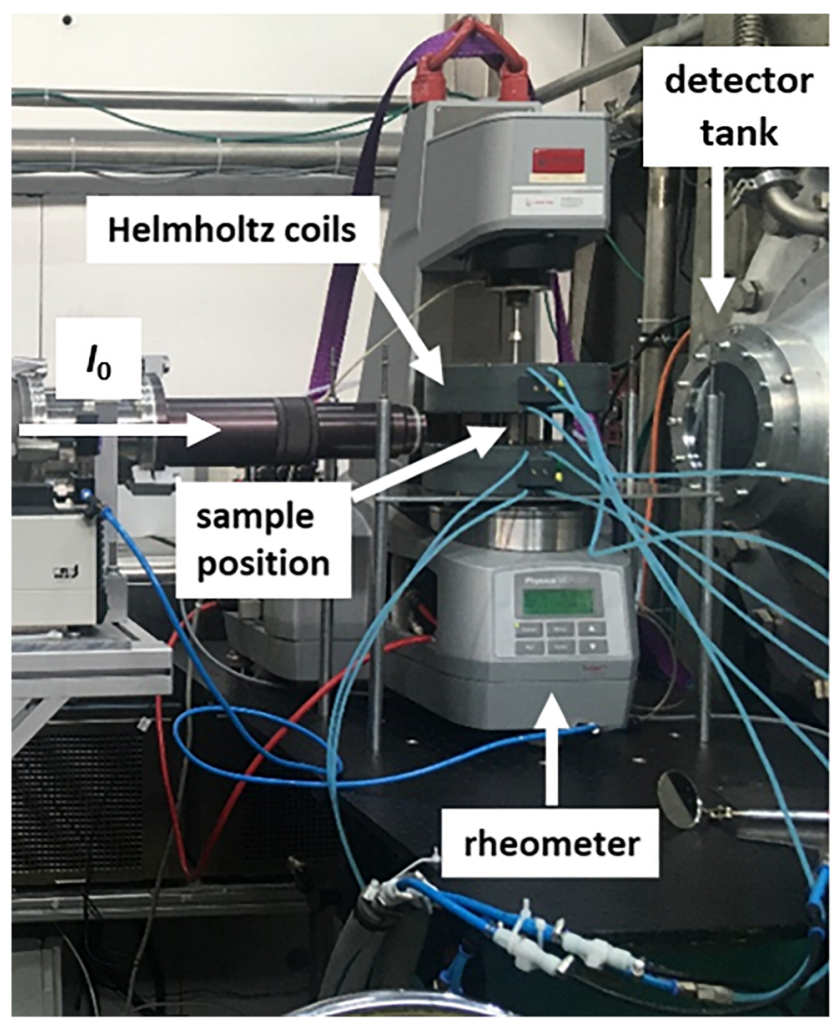

Fig. 2 MagnetoRheoSANS setup at D22 instrument at ILL

rotation axis of the rheometer head. To reach a steady state, the sample was equilibrated for $90 \mathrm{~s}$ at the given shear rate before the shear viscosity and the scattering pattern were recorded using nonpolarized SANS over an exposure time of $10 \mathrm{~min}$. The SANS data were corrected to the transmission, blocked beam, and background of the solvent and empty cup and converted into absolute scale using Grasp [60]. The dependence of the intensity on the azimuthal angle was extracted over a momentum transfer range of $Q=0.019-0.026 \AA^{-1}$.

\section{Results and discussion}

In radial beam configuration with the incident beam parallel to the flow velocity gradient, we observe the shear flow- and magnetic field-induced order-disorder transitions of spindleshaped hematite nanoparticles. The 2D SANS scattering patterns (Figs. 3 and 4) change from an isotropic, circular shape in the absence of magnetic field and shear flow, indicating random orientation of the particles, to a vertically elongated pattern under strong enough fields or shear rates. The magnetic field-dependent SANS in the absence of shear flow is presented in Fig. 3a. In general, a magnetic scattering contribution arising from the nanoparticle magnetization is accessible using magnetic SANS [61]. In the case of the only very weakly ferromagnetic hematite nanospindles, however, the magnetic signal is too small to be detectable by our nonpolarized

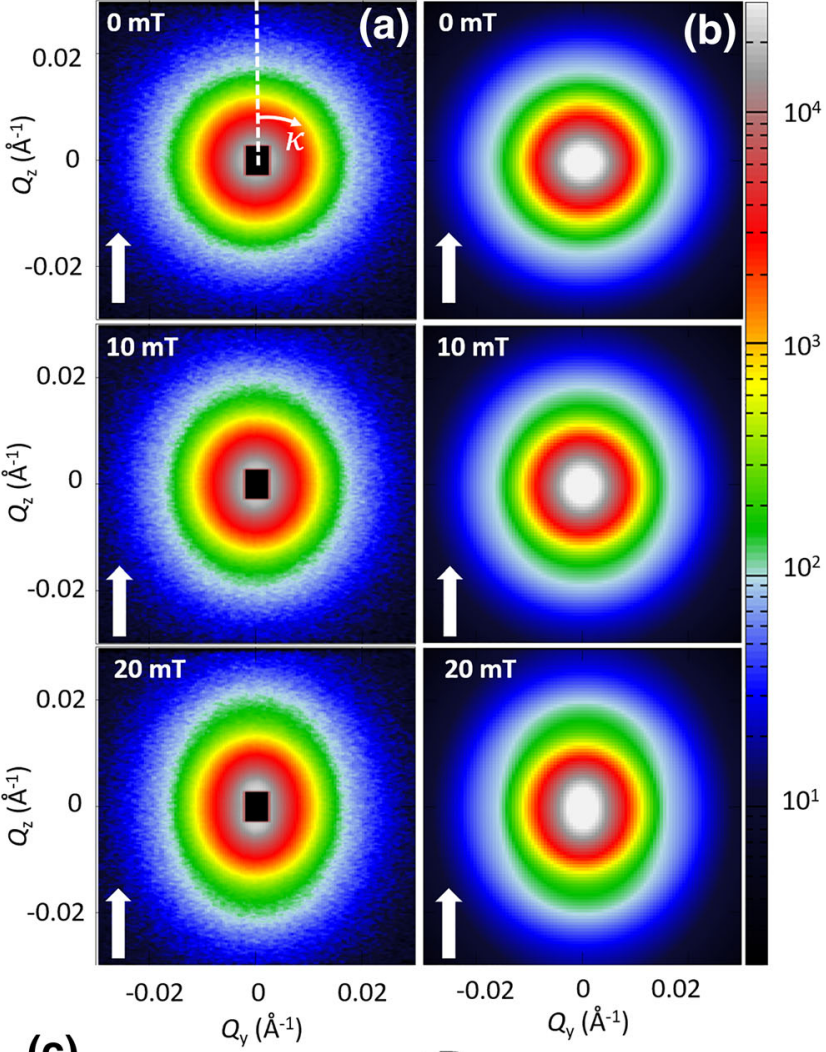

(c)

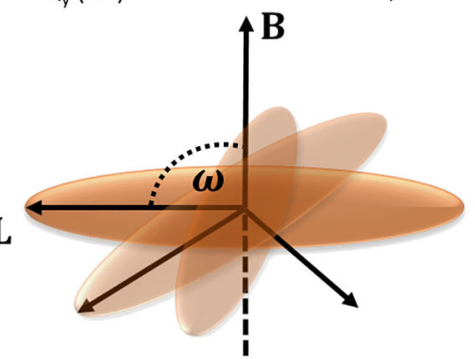

(d)

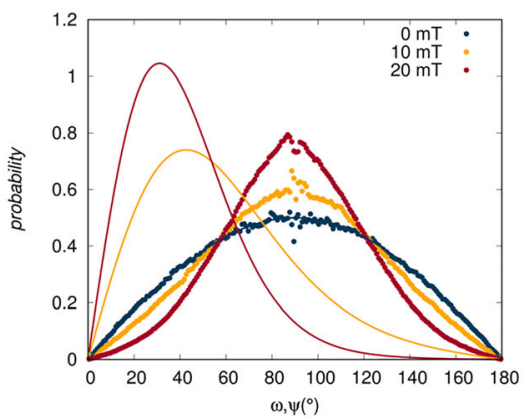

Fig. 3 a 2D scattering cross sections of 3 vol\% of hematite nanospindles under various applied magnetic fields (white arrow indicating the magnetic field direction) and $\mathbf{b}$ simulated $2 \mathrm{D}$ scattering cross sections of rotational ellipsoid. c Sketch of an oriented ellipsoid with length $\mathbf{L}$ free to rotate in the plane perpendicular to the field direction B. d Orientation distribution of the easy axis ( $\psi$, lines) and the resulting orientation distribution of the long spindle axes $\mathbf{L}(\omega$, points) towards the applied magnetic field. Noise observed near $\omega=90^{\circ}$ results from numerical artifacts

setup and is therefore disregarded in this study. The elongation of the scattering cross section in the field direction indicates the preferred orientation of the nanospindles perpendicular to 


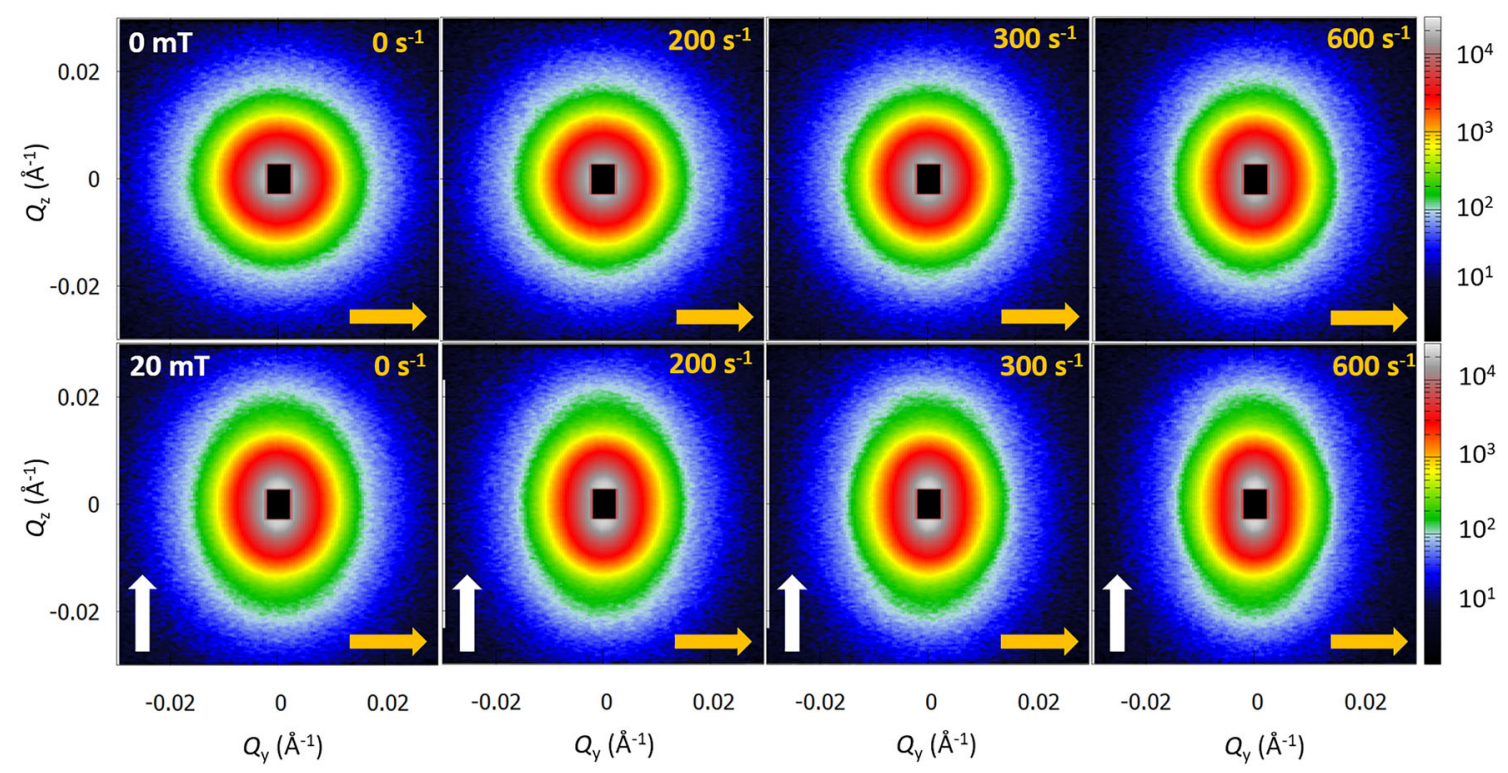

Fig. 4 Evolution of the anisometric 2D scattering at different applied shear rates in applied magnetic fields of $0 \mathrm{mT}$ (top row) and $20 \mathrm{mT}$ (bottom row). Direction and value of shear rates and magnetic field are indicated in orange and white color, respectively

the applied magnetic field. This is in line with our previous studies [30] and is expected as hematite nanospindles are known to bear their magnetic easy axis in the equatorial direction and therefore orient with their principal axis perpendicular to any applied field. If only a magnetic field is applied, the orientation within the normal plane is expected isotropic. Simulations of the field-dependent 2D scattering cross section according to an ellipsoidal form factor with orientation distribution given by Boltzmann statistics [30] are displayed for comparison in Fig. 3b. For the simulation, all morphological (radius, length, and size distribution of length) and magnetic parameters (magnetic particle moment) were fixed as given by the precharacterization. A magnetic field of $20 \mathrm{mT}$ is far below a nominal saturation field, and from magnetization measurements, an average orientation of the magnetic easy axis of $\psi=46.3^{\circ}$ toward the applied magnetic field is expected according to the Langevin function $\mathcal{L}(\xi)=\langle\cos \psi\rangle$ with the Langevin parameter $\xi=\frac{\mu \mu_{0} H}{k_{\mathrm{B}} T}=3.1992$. As a result, the orientation of the long spindle axes is distributed fairly broad around the horizontal plane perpendicular to the field direction ( $\omega=90^{\circ}$, Fig. 3d). The excellent agreement between the measured data and simulations confirms that the coil set provides a homogeneous magnetic field, suitable for SANS measurements in combined magnetic field and shear flow.

In shear flow, an anisometric particle in dispersion rotates and tumbles as it travels in the suspending medium, and in the steady state, a preferred orientation will develop giving rise to scattering anisotropy. Zero-field SANS measurements performed in shear flow reveal increasing scattering anisotropy perpendicular to the shear direction (Fig. 4, top). This feature is attributed to the continuous alignment of the long particle axis in the direction of shear flow.

The application of either magnetic field or shear flow has a very similar effect on the 2D scattering anisotropy, illustrating that the short nanospindle dimension preferentially orients along the vertical direction for both cases. For simultaneous application of flow and magnetic field, an enhanced scattering anisotropy is observed with shear rate, exceeding the
Fig. 5 Field- and sheardependent azimuthal scattering intensity in a $\mathrm{Q}$ range of 0.019 $0.026 \AA^{-1}$. a Field-dependence without applied shear. b Sheardependence in zero magnetic field. c Shear-dependence in $20 \mathrm{mT}$ applied field

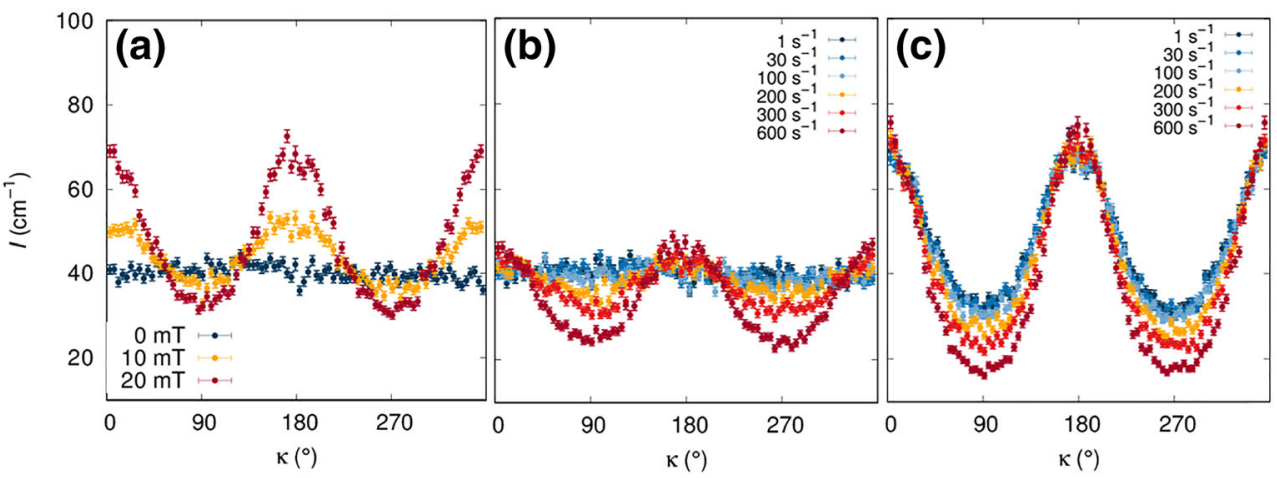


Fig. 6 a Field- and sheardependent scattering anisotropy parameters as extracted from Fig. 5a-c. b Simultaneously measured shear viscosity of the 3 vol\% nanospindles dispersion in water depending on the applied magnetic field
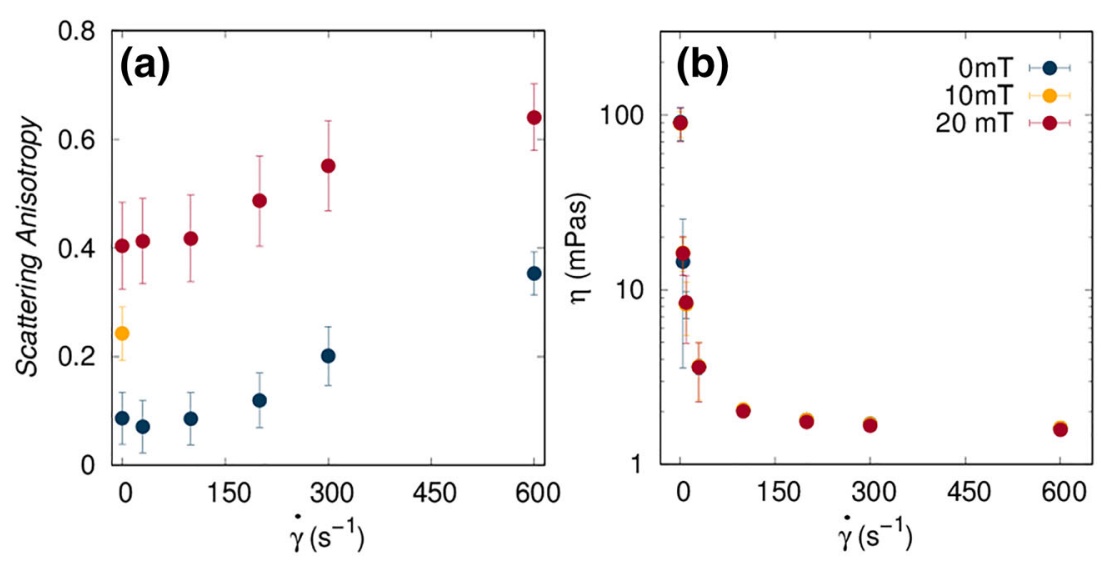

scattering anisotropy observed for field or flow only. The vertical elongation of the scattering cross section is hardly affected, whereas the scattering intensity shifts to lower $Q$ in the horizontal direction indicating a further alignment of the nanospindles (Fig. 4, bottom).

The variation of the scattering intensity with azimuthal angle $\kappa$ illustrates the evolution of the scattering anisotropy with magnetic field and shear flow (Fig. 5). The clearly different effect of magnetic field and shear flow on the scattering anisotropy results from the different orientation behavior induced. Whereas the vertical magnetic field accumulates the spindles in the horizontal plane, the shear deformation takes place mainly within the horizontal plane and aligns the particles with the horizontal shear flow direction. To quantify the scattering anisotropy from the scattering pattern, we determine the anisotropy factor by

$A(Q)=\frac{I\left(Q, 90^{\circ}\right)-I\left(Q, 180^{\circ}\right)}{I\left(Q, 90^{\circ}\right)+I\left(Q, 180^{\circ}\right)}$,

which varies from $A(Q)=0$ for isotropic orientation to $A(Q)=$ 1 for complete alignment of the major axis perpendicular to the magnetic field direction. The anisotropy factor of the SANS pattern increases with magnetic field as well as shear rate (Fig. 6a). Variation of the shear rate for different applied magnetic fields ( $0 \mathrm{mT}$ and $20 \mathrm{mT}$ shown here) leads to a very similar slope of the scattering anisotropy, suggesting a nearly field-independent effect of shear flow on the particle alignment. This is reasonable considering that the modest magnetic field is sufficiently far from saturating the sample and the magnetic torque and the shear flow torque act independently on the particle. Whereas the spindle orientation in the horizontal plane is isotropic in applied magnetic field, shear flow directs the particle orientation into the shear direction and therefore additionally acts on the orientation distribution within the horizontal plane.

The shear viscosity measured simultaneously to the SANS experiment is presented in Fig. 6b. In contrast to the SANS experiment, it does not exhibit a significant magnetic field dependence. A shear thinning effect is observed for small shear rates that might be related to initial orientation of the spindles in the dilute dispersion. With increasing shear rate, only the behavior of the solvent contributes to the viscosity. This observation is in line with expectations for a dilute nanospindle dispersion far below the critical interaction concentration, where field- and shear-induced orientations of the nanoparticles have no significant effect on the shear viscosity. The absence of a visible magnetic field effect confirms in this case the successful shielding of the rheometer against the imposed magnetic field. At the same time, SANS is very sensitive to the nanoparticle orientation even if a rheological effect is absent.

The combination of rheology in applied magnetic field and SANS to MagnetoRheoSANS thus gives access to clearly distinguishable effects of magnetic field and shear flow on the nanoparticle orientation behavior. A more detailed, quantitative description will require in-depth simulation and modeling of the field- and shear flow-induced orientation distributions, which is beyond the scope of this paper.

\section{Conclusion}

Small-angle scattering is a valuable tool for studying flowing systems of dispersed nanoparticles to reveal the collective orientation distribution of particles and the relation between microscopic organization and macroscopic rheological properties. In this work, a magnetorheology setup available for SANS is presented, which extends the available RheoSANS suite at the ILL toward the application of magnetic field and gives the opportunity of polarized neutron scattering. Measurements in radial and tangential scattering geometry through the rheology setup allow reconstructing the 3D orientational distribution and intraparticle arrangement. It opens the opportunity to investigate the magnetic field- and flowinduced structure formations, and hence the microscopic origin of magnetoviscous effects in magnetically functionalized soft matter systems. The sample environment is available for the user community of ILL and is of interest for the study of 
complex magnetic fluids like magnetic gels, elastomers, and ferromagnetic liquid crystals.

As a model system, the alignment of anisometric particles within the fluid under the influence of shear and magnetic field is presented. A detailed determination of the order parameter and the use of a dedicated particle model in the future will be necessary for a quantitative description of the particle orientation dynamics under combined shear and magnetic field.

Acknowledgments We are grateful to the technical workshop of the Institute of Physical Chemistry at University of Cologne, headed by V. Klippert, who constructed the magnetic coil sets.

We thank the Institut Laue-Langevin for provision of neutron beam time at D22.

Availability of data The SANS data is stored and will be available based on the ILL data policy (https://doi.ill.fr/10.5291/ILL-DATA.5-32-785).

Funding Open Access funding provided by Projekt DEAL. This research was supported by Deutsche Forschungsgemeinschaft (DFG, project DI 1788/2-1) as well as by a PhD fellowship (D.Z.) and the internship program (K.G.) supported by the Institut Laue-Langevin.

\section{Compliance with ethical standards}

Conflict of interest The authors declare that they have no conflict of interest.

Open Access This article is licensed under a Creative Commons Attribution 4.0 International License, which permits use, sharing, adaptation, distribution and reproduction in any medium or format, as long as you give appropriate credit to the original author(s) and the source, provide a link to the Creative Commons licence, and indicate if changes were made. The images or other third party material in this article are included in the article's Creative Commons licence, unless indicated otherwise in a credit line to the material. If material is not included in the article's Creative Commons licence and your intended use is not permitted by statutory regulation or exceeds the permitted use, you will need to obtain permission directly from the copyright holder. To view a copy of this licence, visit http://creativecommons.org/licenses/by/4.0/.

\section{References}

1. Rodríguez-Arco L, López-López MT, Zubarev AY, Gdula K, Durán JDG (2014) Inverse magnetorheological fluids. Soft Matter 10:6256-6265

2. Ramos J, Klingenberg DJ, Hidalgo-Alvarez R, de Vicente J (2011) Steady shear magnetorheology of inverse ferrofluids. J Rheol (N Y N Y) 55:127-152

3. Saldivar-Guerrero R, Richter R, Rehberg I, Aksel N, Heymann L, Rodriguez-Fernández OS (2006) Viscoelasticity of mono- and polydisperse inverse ferrofluids. J Chem Phys 125:084907

4. Zubarev A, Bonhome-Espinosa AB, Alaminos M, Duran JDG, Lopez-Lopez MT (2019) Rheological properties of magnetic biogels. Arch Appl Mech 89:91-103

5. Gila-Vilchez C, Bonhome-Espinosa AB, Kuzhir P, Zubarev A, Duran JDG, Lopez-Lopez MT (2018) Rheology of magnetic alginate hydrogels. J Rheol (N Y N Y) 62:1083-1096
6. Bonhome-Espinosa AB, Campos F, Rodriguez IA, Carriel V, Marins JA, Zubarev A, Duran JDG, Lopez-Lopez MT (2017) Effect of particle concentration on the microstructural and macromechanical properties of biocompatible magnetic hydrogels. Soft Matter 13:2928-2941

7. Ilg P (2013) Stimuli-responsive hydrogels cross-linked by magnetic nanoparticles. Soft Matter 9:3465-3468

8. Messing R, Schmidt AM (2011) Perspectives for the mechanical manipulation of hybrid hydrogels. Polym Chem 2:18-32

9. Roeben E, Roeder L, Messing R, Frickel N, Marten G, Gelbrich T, Schmidt AM (2013). Intell Hydrogels 140:131-148

10. Kordonski WI, Jacobs SD (1996) Magnetorheological finishing. Int J Mod Phys B 10:2837-2848

11. Urreta H, Leicht Z, Sanchez A, Agirre A, Kuzhir P, Magnac G (2010) Hydrodynamic bearing lubricated with magnetic fluids. J Intell Mater Syst Struct 21:1491-1499

12. Lum GZ, Ye Z, Dong X, Marvi H, Erin O, Hu W, Sitti M (2016) Shape-programmable magnetic soft matter. Proc Natl Acad Sci U S A 113:E6007-E6015

13. Lopez-Lopez MT, Scionti G, Oliveira AC, Duran JDG, Campos A, Alaminos M, Rodriguez IA (2015) Generation and characterization of novel magnetic field-responsive biomaterials. PLoS One 10:117

14. Wu J, Gong X, Fan Y, Xia H (2011) Physically crosslinked poly(vinyl alcohol) hydrogels with magnetic field controlled modulus. Soft Matter 7:6205-6212

15. Zhao X, Kim J, Cezar CA, Huebsch N, Lee K, Bouhadir K, Mooney DJ (2011) Active scaffolds for on-demand drug and cell delivery. Proc Natl Acad Sci U S A 108:67-72

16. Potisk T, Pleiner H, Svenšek D, Brand HR (2018) Effects of flow on the dynamics of a ferromagnetic nematic liquid crystal. Phys Rev E 97:1-13

17. Mertelj A, Lisjak D, Drofenik M, Čopič M (2013) Ferromagnetism in suspensions of magnetic platelets in liquid crystal. Nature 504: 237-241

18. Mertelj A, Lisjak D (2017) Ferromagnetic nematic liquid crystals. Liq Cryst Rev 5:1-33

19. Rupnik PM, Lisjak D, Čopič M, Čopar S, Mertelj A (2017) Fieldcontrolled structures in ferromagnetic cholesteric liquid crystals. Sci Adv 3:1-11

20. Dhar P, Cao Y, Fischer TM, Zasadzinski JA (2010) Active interfacial shear microrheology of aging protein films. Phys Rev Lett 104: $1-4$

21. Bender P, Tschöpe A, Birringer R (2014) Magnetization measurements reveal the local shear stiffness of hydrogels probed by ferromagnetic nanorods. J Magn Magn Mater 372:187-194

22. Günther A, Bender P, Tschöpe A, Birringer R (2011) Rotational diffusion of magnetic nickel nanorods in colloidal dispersions. $\mathrm{J}$ Phys Condens Matter 23:325103

23. Klein T, Laptev A, Günther A, Bender P, Tschöpe A, Birringer R (2009) Magnetic-field-dependent optical transmission of nickel nanorod colloidal dispersions. J Appl Phys 106:114301

24. Martchenko I, Dietsch H, Moitzi C, Schurtenberger P (2011) Hydrodynamic properties of magnetic nanoparticles with tunable shape anisotropy: prediction and experimental verification. J Phys Chem B 115:14838-14845

25. Eliseev AA, Eliseev AA, Trusov LA, Chumakov AP, Boesecke P, Anokhin EO, Vasiliev AV, Sleptsova AE, Gorbachev EA, Korolev VV, Kazin PE (2018) Rotational dynamics of colloidal hexaferrite nanoplates. Appl Phys Lett 113:113106

26. Glinka C, Bleuel M, Tsai P, Zákutná D, Honecker D, Dresen D, Mees F, Disch S (2020) Sub-millisecond time-resolved small-angle neutron scattering measurements at NIST. J Appl Crystallogr 53: 598-604

27. Shliomis MI (1972) Effective viscosity of magnetic fluids. Sov Phys JETP 34:1291-1294 
28. Jeffery GB (1922) The motion of ellipsoidal particles immersed in a viscous fluid. Proc R Soc London Ser A 102:161-179

29. Tschöpe A, Birster K, Trapp B, Bender P, Birringer R (2014) Nanoscale rheometry of viscoelastic soft matter by oscillating field magneto-optical transmission using ferromagnetic nanorod colloidal probes. J Appl Phys 116:184305

30. Zákutná D, Falke Y, Dresen D, Prévost S, Bender P, Honecker D, Disch S (2019) Morphological and crystallographic orientation of hematite spindles in an applied magnetic field. Nanoscale 11:71497156

31. Hoffelner D, Kundt M, Schmidt AM, Kentzinger E, Bender P, Disch S (2015) Directing the orientational alignment of anisotropic magnetic nanoparticles using dynamic magnetic fields. Faraday Discuss 181:449-461

32. Reufer M, Dietsch H, Gasser U, Hirt A, Menzel A, Schurtenberger P (2010) Morphology and orientational behavior of silica-coated spindle-type hematite particles in a magnetic field probed by small-angle X-ray scattering. J Phys Chem B 114:4763-4769

33. Reufer M, Martinez VA, Schurtenberger P, Poon WCK (2012) Differential dynamic microscopy for anisotropic colloidal dynamics. Langmuir 28:4618-4624

34. Nack A, Seifert J, Passow C, Wagner J (2018) Hindered nematic alignment of hematite spindles in poly( $\mathrm{N}$-isopropylacrylamide) hydrogels: a small-angle X-ray scattering and rheology study: A. J Appl Crystallogr 51:87-96

35. Bender P, Günther A, Honecker D, Wiedenmann A, Disch S, Tschöpe A, Michels A, Birringer R (2015) Excitation of Ni nanorod colloids in oscillating magnetic fields: a new approach for nanosensing investigated by TISANE. Nanoscale 7:17122-17130

36. Comesaña-Hermo M, Ciuculescu D, Li ZA, Stienen S, Spasova M, Farle M, Amiens C (2012) Stable single domain co nanodisks: synthesis, structure and magnetism. J Mater Chem 22:8043-8047

37. Satoh A, Cuadra R (2019) Experimental verification of negative magnetorheological characteristics in spindle-like hematite particle suspensions. J Magn Magn Mater 469:606-612

38. Martin JJ, Riederer MS, Krebs MD, Erb RM (2015) Understanding and overcoming shear alignment of fibers during extrusion. Soft Matter 11:400-405

39. Wiedenmann A, Hoell A, Kammel M, Boesecke P (2003) Fieldinduced pseudocrystalline ordering in concentrated ferrofluids. Phys Rev E 68:031203

40. Taheri SM, Michaelis M, Friedrich T et al (2015) Self-assembly of smallest magnetic particles. Proc Natl Acad Sci U S A 112:1448414489

41. Odenbach S (2004) Recent progress in magnetic fluid research. J Phys Condens Matter 16:R1135-R1150

42. Park BJ, Fang FF, Choi HJ (2010) Magnetorheology: materials and application. Soft Matter 6:5246-5253

43. Wolff M, Kuhns P, Liesche G, Ankner JF, Browning JF, Gutfreund P (2013) Combined neutron reflectometry and rheology. J Appl Crystallogr 46:1729-1733

44. Maranzano BJ, Wagner NJ (2002) Flow-small angle neutron scattering measurements of colloidal dispersion microstructure evolution through the shear thickening transition. J Chem Phys 117: 10291-10302

45. Velichko E, Tian B, Nikolaeva T, Koning J, van Duynhoven J, Bouwman WG (2019) A versatile shear cell for investigation of structure of food materials under shear. Colloids Surfaces A Physicochem Eng Asp 566:21-28

46. Stellamanns E, Meissner D, Lohmann M, Struth B (2013) A unique rheology / SAXS combination at DESY / Petra III. J Phys Conf Ser 425:9-12

47. Porcar L, Pozzo D, Langenbucher G, Moyer J, Butler PD (2011) Rheo-small-angle neutron scattering at the National Institute of Standards and Technology Center for Neutron Research. Rev Sci Instrum 82:1-8

48. Eberle APR, Porcar L (2012) Flow-SANS and Rheo-SANS applied to soft matter. Curr Opin Colloid Interface Sci 17:33-43

49. Panine P, Gradzielski M, Narayanan T (2003) Combined rheometry and small-angle $\mathrm{X}$-ray scattering. Rev Sci Instrum 74:2451-2455

50. Mock EB, Zukoski CF (2007) Investigating microstructure of concentrated suspensions of anisotropic particles under shear by small angle neutron scattering. J Rheol (N Y N Y) 51:541-559

51. Lehmkühler F, Steinke I, Schroer MA, Fischer B, Sprung M, Grübel G (2017) Microsecond structural rheology. J Phys Chem Lett 8:3581-3585

52. Jaksch S, Holderer O, Gvaramia M, Ohl M, Monkenbusch M, Frielinghaus H (2017) Nanoscale rheology at solid-complex fluid interfaces. Sci Rep 7:1-7

53. Krishnamurthy VV, Bhandar AS, Piao M, Zoto I, Lane AM, Nikles DE, Wiest JM, Mankey GJ, Porcar L, Glinka CJ (2003) Shear- and magnetic-field-induced ordering in magnetic nanoparticle dispersion from small-angle neutron scattering. Phys Rev E Stat Physics, Plasmas, Fluids, Relat Interdiscip Top 67:6

54. Krishnamurthy VV, Mankey GJ, He B, Piao M, Wiest JM, Nikles DE, Porcar L, Robertson JL (2008) Orientational distributions and nematic order of rodlike magnetic nanoparticles in dispersions. Phys Rev E - Stat Nonlinear, Soft Matter Phys 77:1-8

55. Pop LM, Hilljegerdes J, Odenbach S, Wiedenmann A (2004) The microstructure of ferrofluids and their rheological properties. Appl Organomet Chem 18:523-528

56. Pop LM, Odenbach S (2006) Investigation of the microscopic reason for the magnetoviscous effect in ferrofluids studied by small angle neutron scattering. J Phys Condens Matter 18:S2785-S2802

57. Pop LM, Odenbach S, Wiedenmann A, Matoussevitch N, Bönnemann H (2005) Microstructure and rheology of ferrofluids. J Magn Magn Mater 289:303-306

58. Ozaki M, Kratohvil S, Matijević E (1984) Formation of monodispersed spindle-type hematite particles. J Colloid Interface Sci 102:146-151

59. Disch S, Graef K, Zákutná D, Honecker D, Keiderling U, Porcar L, Wiedenmann A (2013) Inst Laue-Langevin. https://doi.org/10. 5291/ILL-DATA.5-32-785

60. Dewhurst CD, Grasp, available online at https://www.ill.eu/users/ support-labs-infrastructure/software-scientific-tools/grasp/

61. Mühlbauer S, Honecker D, Périgo ÉA, Bergner F, Disch S, Heinemann A, Erokhin S, Berkov D, Leighton C, Eskildsen MR, Michels A (2019) Magnetic small-angle neutron scattering. Rev Mod Phys 91:015004

Publisher's note Springer Nature remains neutral with regard to jurisdictional claims in published maps and institutional affiliations. 